\title{
Nose to brain delivery in rats: effect of surface charge of rhodamine B labeled nanocarriers on brain subregion localization
}

A. Bonaccorso ${ }^{1}$, T. Musumeci ${ }^{1 *}$, M.F. Serapide ${ }^{2}$, R. Pellitteri ${ }^{3}$, I. F. Uchegbu ${ }^{4}$, G. Puglisi ${ }^{1}$

${ }^{1}$ Laboratory of Drug delivery Technology- Department of Drug Science, University of Catania, Via S. Sofia, 64-95125. Catania, Italy.

${ }^{2}$ Department of Biomedical and Biotechnological Science- University of Catania, Via S. Sofia, 6495125, Catania, Italy.

${ }^{3}$ Institute of Neurological Sciences, CNR, Section of Catania, Via P. Gaifami 18 - 95126 Catania, Italy.

${ }^{4}$ UCL, School of Pharmacy, University College London, 29_39 Brunswick Square, London WC1N 1AX, United Kingdom.

Corresponding author*

Teresa Musumeci, PhD

Department of Drug Sciences, University of Catania

Città Universitaria, Via S. Sofia, 64; I-95125 Catania, Italy

Email: teresa.musumeci@unict.it

phone: +39095 7384021 


\begin{abstract}
Nose to brain delivery and nanotechnology are the combination of innovative strategies for molecules to reach the brain and to bypass blood brain barriers. In this work we investigated the fate of two rhodamine B labeled polymeric nanoparticles (Z-ave $<250 \mathrm{~nm}$ ) of opposite surface charge in different areas of the brain after intranasal administration in rats. A preliminary screening was carried out to select the suitable positive (chitosan/poly-l-lactide-co-glycolide) nanocarrier through Photon Correlation Spectroscopy and Turbiscan. Physico-chemical and technological characterizations of poly-l-lactide-co-glycolide (negative) and chitosan/poly-l-lactide-co-glycolide (positive) fluorescent labeled nanoparticles were performed. The animals were allocated to three groups receiving negative and positive polymeric nanoparticles via single intranasal administration or no treatment. The localization of both nanocarriers in different brain areas was detected using fluorescent microscopy. Our data revealed that both nanocarriers reach the brain and are able to persist in the brain up to $48 \mathrm{~h}$ after intranasal administration. Surface charge influenced the involved pathways in their translocation from the nasal cavity to the central nervous system. The positive charge of nanoparticles slows down brain reaching and the trigeminal pathway is involved, while the olfactory pathway may be responsible for the transport of negatively charged nanoparticles, and systemic pathways are not excluded.
\end{abstract}

Keywords: intranasal delivery, chitosan nanoparticles, PLGA, brain, fluorescent microscopy, in vivo study, rats. 


\section{Abbreviations:}

BBB- Blood Brain Barrier

CNS- Central Nervous System

CS- Chitosan

CSF-Cerebral spinal fluid

DSC- Differential Scanning Calorimetry

$\%$ DTE- drug targeting efficiency percentage

\%DTP- direct transport percentage

E.E. - Encapsulation Efficiency

FDA- Food and Drug Administration

GRAS- Generally Recognized As Safe

IN- IntraNasal

LMW- Low Molecular Weight

MPS- Mononuclear phagocyte system

N2B- Nose to Brain

NPA- PLGA NPs

NPBC-chitosan/PLGA NPs (NPBC3; NPBC5; NPBC10; NPBC20)

NPs- nanoparticles

P.D.I.-polidispersity index

PBS- Phosphate Buffer Solution

PCS- Photon Correlation Spectroscopy

PFA-Paraformaldehyde

PLA- Poly-lactide

PLGA- Poly-lactide-co-glycolide

SEM- Scanning electron microscopy

TSI- Turbiscan stability index 
ZP- Zeta potential values

$\Delta \mathrm{H}$ - enthalpy changes

$\triangle \mathrm{BS}$ - Delta Backscattering

$\Delta \mathrm{T}$ - Delta Transmission 


\section{Introduction}

The intranasal (IN) route is commonly used for local disease treatments and systemic delivery of drugs; furthermore, this route is considered to be a potential alternative way of administrating drugs to the brain, specifically to bypass the blood-brain barrier (BBB). The nose to brain route serves as a direct delivery route of molecules to the brain (Chapman et al., 2013). Novel delivery devices have been developed for this purpose (ViaNase ID ${ }^{\mathrm{TM}}$; OptiNose; POD-Precision Olfactory Delivery-device) (Djupesland et al., 2014). These devices can transport nanosuspensions or molecules in solution to specific regions of the nasal cavity in man (olfactory region).

IN administration is a non-invasive method and could represent an alternative strategy for drugs commonly administered orally or intravenously and that have difficulty moving across the BBB (Chapman et al., 2013; Tosi et al., 2016). As reported by Kozlovskaya et al. (2014), several papers describe drug accumulation in the brain following IN application of the drug solution or suspension, but some free drugs could achieve low therapeutic concentration in the brain using this route, as previously demonstrated (Piazza et al., 2014, Meredith et al., 2015, Zhang et al, 2014). Among the different types of drug carriers, due to their mean size, nanoparticles (NPs) used for drug delivery, may increase the therapeutic dose of drugs in the brain when administered intranasally. In fact, these carriers could protect against local enzymatic degradation, increase IN residence time by avoiding mucociliary clearance, and possibly achieve prolonged release at the target site. Shadab et al. (2014) have shown that NPs transported drugs into the brain suggesting a direct transport to this site that bypasses the BBB (Shadab et al., 2014, Kozlovskaya et al., 2014).

The selection of polymers requires a preliminary evaluation related to the site of administration and the hypothetical fate of NPs (Kumari et al., 2010). Chitosan (CS) NPs are the most commonly investigated nanocarriers for nose to brain delivery and are suitable for the transport of hydrophilic molecules (Casettari et al., 2014). CS is a natural polysaccharide that is capable of increasing the NPs residence time in the nares due to its mucoadhesive properties and this polymer may also act as 
a penetration enhancer; furthermore, it is generally recognized as safe material (GRAS) (Charlton et al., 2007; Duttagupta et al., 2015). Currently, hybrid nanocarriers are prepared using two different types of polymers, and chitosan could be used to confer a positive charge to a PLGA nanoparticle surface (Bharali et al. 2015). Poly-lactide-co-glycolide (PLGA), co-polymer of poly-lactide (PLA), is a suitable material to prepare NPs for lipophilic or amphiphilic molecules for its tunable physicochemical properties and it is a Food and Drug Administration (FDA) approved polymer (Kapoor et al., 2015).

In our previous in vitro study, we demonstrated the influence of surface charge on the uptake of rhodamine B labeled NPs via olfactory glial cells (Musumeci et al., 2014), according to other authors for a different type of cells.

The proof of concept of nose to brain delivery of NPs is claimed by different authors; for example, some described toxicological effects in the brain because of inhaled ultrafine particles (van Berlo et al., 2014). Others demonstrated the nose to brain direct transport through the evaluation of the "drug targeting efficiency percentage (\%DTE)" or "direct transport percentage (\%DTP)" values or through in vivo behavioral studies. In particular, authors compared the results obtained after IN administration of the drug loaded NPs and other routes (Kozlovskaya et al., 2014; Phukan et al., 2016).

Despite the promising results, some questions have not been answered. First of all, is there a tropism for particular brain sub-regions after IN administration of NPs? If so, is this tropism influenced by anatomical-physiological conditions? Could surface properties of NPs influence the localization in different brain sub-regions? Literature data show that NPs reach the brain prevalently in the olfactory and/or trigeminal region after IN administration, but no correlation was made between these results and the surface properties of NPs investigated during a time course.

In this work, we carried out in vivo studies on rats in order to correlate opposite surface (positive and negative) charges of the selected rhodamine B labeled NPs with the regional brain localization after IN administration up to $48 \mathrm{~h}$. Chitosan-PLGA (poly(lactic-co-glycolic acid) NPs were selected 
after a preliminary screening using photon correlation spectroscopy (PCS) and Turbiscan analyses. In vivo brain localization analyses were carried out in healthy rats after IN administration of PLGA and Chitosan-PLGA fluorescent NPs through fluorescent microscopy.

\section{Materials and methods}

\section{Materials}

Low molecular weight (LMW: molecular weight is 50,000-190,000 daltons based on viscosity; viscosity 20-300 cps, deacetylation degree 75-85\%) water-soluble chitosan derived from crab shells, Tween ${ }^{\circledR} 80$, rhodamine Band glacial acetic acid $\left(\mathrm{d} 1,049 \mathrm{~g} / \mathrm{ml}\right.$ a $\left.25^{\circ} \mathrm{C}\right)$ were purchased from Sigma-Aldrich (Milan, Italy). Sodium sulfate, sodium hydroxide and all other chemicals were

analytical grade, purchased from Analyticals, Carlo Erba. The polymer Resomer ${ }^{\circledR} 502$ H poly-(D,Llactide-co-glycolide) (50:50, molecular weight is 30,000-60,000) were purchased from Boehringer Ingelheim Pharma GmbH\&Co. KG (Boehringer Ingelheim, Germany). Ultrapure water was used throughout this study. For biological studies, we used adult male Wistar rats (200-250 gr b.w., Envigo s.r.l. - ex Harlan, Italy), Zoletil 100 and Dexdomitor anesthetics, paraformaldehyde and other chemicals from Sigma-Aldrich (Milan, Italy).

\section{PLGA nanoparticle preparation as negative surface charge nanocarriers}

PLGA NPs (NPA) were prepared using the solvent displacement method followed by polymer deposition as previously reported (Musumeci et al., 2013). Briefly, the chosen polymer (75 mg) was dissolved in acetone $(20 \mathrm{ml})$. The organic phase was added drop by drop to a $40 \mathrm{ml}$ water/ethanol solution $(1: 1, \mathrm{v} / \mathrm{v})$ containing $0.5 \%(\mathrm{w} / \mathrm{v})$ Tween ${ }^{\circledR} 80$ under magnetic stirring, obtaining a milky colloidal suspension. The organic solvent was then evaporated off under high vacuum at $40^{\circ} \mathrm{C}$. Fluorescent-labeled NPs were prepared by co-dissolving rhodamine B $(5 \mu \mathrm{g} / \mathrm{ml})$ with the polymer in the organic phase. The different formulations were purified through ultracentrifugation 
(15000×g) for $1 \mathrm{~h}$ at $10^{\circ} \mathrm{C}$, using a Beckman (Fullerton, CA) J2-21 model centrifuge equipped with a Beckman JA-20.01 fixed-angle rotor. After washing, the obtained NPs were re-suspended in $5 \mathrm{ml}$ of filtered water $(0.22 \mu \mathrm{m}$ Sartorius membrane filters $)$. This procedure was repeated three times. The obtained samples were characterized according to mean size, size distribution and surface chemistry.

\section{Chitosan/PLGA nanoparticle preparation as surface charge positive nanocarriers}

Chitosan/PLGA NPs were prepared using the nanoprecipitation method, "In situ coating method",as described by Sanna et al. (2012) with little modification (modified by Sanna et al., 2012). Chitosan/PLGA NPs, identified as NPBC, were prepared. The influence of the amount of polymer was evaluated on physico-chemical characteristics. Briefly, PLGA at different concentrations (3, 5, 10, $20 \mathrm{mg} / \mathrm{ml}$; respectively identified as NPBC3, NPBC5, NPBC10, NPBC20) was dissolved in acetone and was added dropwise into a chitosan (CS) solution $(0.1 \% \mathrm{w} / \mathrm{v}$ solubilzed in acetic acid solution $0.1 \% \mathrm{v} / \mathrm{v}, 5 \mathrm{~mL}$ ). The resulting milky colloidal suspension was evaporated at room temperature to remove a portion of the organic solvent $(1 \mathrm{~h})$. Then the organic solvent was evaporated off under high vacuum at $40^{\circ} \mathrm{C}$. The different formulations were purified through ultracentrifugation $\left(11000 \times \mathrm{g}\right.$ ) for $1 \mathrm{~h}$ at $8{ }^{\circ} \mathrm{C}$, using a Beckman (Fullerton, CA) J2-21 model centrifuge equipped with a Beckman JA-20.01 fixed-angle rotor. After washing, the obtained NPs were re-suspended in $5 \mathrm{ml}$ of filtered water $(0.22 \mu \mathrm{m}$ Sartorius membrane filters). This procedure was repeated three times. The obtained samples were characterized according to mean size, size distribution and surface chemistry. Also Turbiscan analysis was carried out. After the selection of the suitable nanosuspension (NPBC3), fluorescent-labeled Chitosan/PLGA NPs were prepared by co-dissolving rhodamine B $(5 \mu \mathrm{g} / \mathrm{mL})$ with the PLGA polymer in the organic phase.

\section{Particle size, size distribution and Zeta potential analyses}

The particle size and the polidispersity index (P.D.I.) of nanoaggregates were measured performing photon correlation spectroscopy (PCS) with a Zetasizer Nano S90 (Malvern Instruments, Malvern, 
UK) at a detection angle of $90^{\circ}$, at $25^{\circ} \mathrm{C}$ with a $4 \mathrm{~mW}$ He-Ne laser operating at $633 \mathrm{~nm}$. Each value was measured in triplicate. The results are shown as mean \pm standard deviation. The samples were analyzed using a disposable cuvette, "DTS 0012 Disposable sizing cuvette", withdrawing $700 \mu 1$ of suspension. The Zeta potential values (ZP), which reflect the electric charge on the particle surface, were determined at $25^{\circ} \mathrm{C}$ using the same equipment described previously. For the measurement, samples were diluted appropriately with ultra-purified water.

\section{Turbiscan study}

Turbiscan $^{\mathrm{TM}}$ AGS (Formulaction, l'Union, France, a robot and a storage station integrated TurbiscanLAB) was used to examine the dispersion stability of NPs. This instrument allows for the use of a simple technique to observe reversible (creaming and sedimentation due to fluctuation on particle size and volume) and irreversible (coalescence and segregation due to particle size variation) destabilization phenomena in the sample. Turbiscan ${ }^{\mathrm{TM}} \mathrm{AGS}$ is useful to detect destabilization phenomena much earlier and also in a more simple way than other methods. Each suspension $(15 \mathrm{~mL})$ was placed in flat-bottomed cylindrical glass tubes, which were placed in the instrument and the transmission of light from the suspensions was then measured periodically (1h) along the height at room temperature and at $25^{\circ} \mathrm{C}$ for up to $48 \mathrm{~h}$. The sedimentation behavior of the suspensions was monitored by measuring the backscattering and monochromatic transmission near infrared $(\lambda=850 / 880 \mathrm{~nm})$. The sedimentation rate was evaluated from the change in backscattering intensity at the top portion of the sample.

Physico-chemical, morphological, calorimetric analyses and in vitro release study of labeled NPs

Differential scanning calorimetric analysis

A Mettler Toledo DSC 1 STAR $^{\mathrm{e}}$ system equipped with a PolyScience temperature controller (PolyScienceIllinois, USA) was used to perform calorimetric analyses. The detection system was a 
HSS8 high sensitivity sensor (120 gold-gold/palladium-palladium thermocouples) and the ceramic sensor (Mettler Full Range; FRS5) with 56 thermocouples. The signal time constant was $18 \mathrm{~s}$ and the digital resolution of the measurement signal was less than $0.04 \mu \mathrm{W}$. Calorimetric resolution and sensitivity, determined through the TAWN test, is respectively 0.12 and 11.9 . The sampling rate is 50 values/second. The sensitivity was automatically chosen as the maximum possible through the calorimetric system, and the reference was an empty pan. The calorimetric system was calibrated, in temperature and enthalpy changes, by using indium by following the procedure of the DSC 1 Mettler TA STAR ${ }^{\mathrm{e}}$ instrument. NPs were sealed in an aluminum pan and submitted to DSC analysis to determinate the influence of coating on thermotropic parameters of NPs. Each sample was submitted to heating and cooling cycles in the temperature range $10-200{ }^{\circ} \mathrm{C}$ at a scanning rate of $5^{\circ} \mathrm{C} / \mathrm{min}$ (heating) and at a scanning rate of $10^{\circ} \mathrm{C} / \mathrm{min}$ (cooling). Transition temperature and enthalpy changes $(\Delta \mathrm{H})$ were calculated from peak areas with the MettlerSTAR ${ }^{\mathrm{e}}$ Evaluation software system (version 13.00) installed on Optiplex 3020 DELL.

\section{SEM analysis}

Scanning electron microscopy (SEM) was performed to evaluate the surface morphology of NPs using a SEM Philips mod 500. NP samples were dried for $24 \mathrm{~h}$ before the analysis. A small amount of NPs was stuck on a double-sided tape attached on a metallic sample stand, then coated, under argon atmosphere, with a thin layer of gold, using a POLARON E5100 SEM Coating Unit.

\section{In vitro release studies}

The in vitro release studies of rhodamine from PLGA NPs and chitosan/PLGA NPs were performed using cellulose membrane dialysis tubing (Spectra/Por® 3 Dialysis Membranes, MWCO 3.5kD, Flat width $18 \mathrm{~mm}$, diameter $11.5 \mathrm{~mm}$, Vol/Lg $1.1 \mathrm{ml} / \mathrm{cm}$, Length $15 \mathrm{~m} / 50 \mathrm{ft}$; Spectrum ${ }^{\circledR}$ Laboratories). The samples $(1 \mathrm{ml})$ were enclosed in dialysis bags and incubated in $19 \mathrm{ml} \mathrm{PBS}(\mathrm{pH}$ 7.4; $\mathrm{pH} 5.8$ ) with constant shaking, $100 \mathrm{rpm}$, in water bath at $37 \pm 0.5{ }^{\circ} \mathrm{C}$. At predetermined time intervals $(1,2,3,4,5,24,48 \mathrm{~h})$, a fixed volume of release medium was withdrawn and replaced 
with an equivalent amount of release media. These samples were analyzed using UV spectrophotometry at rhodamine $\lambda$-max (555.2 $\mathrm{nm}$ in PBS pH7.4; and $\mathrm{pH} 5.8$ ) (UV-VIS 1601 spectrophotometer, Shimadzu Italia, Milan, Italy). Calibration curves for the quantitative evaluation of the probe were linear in the following ranges:(i) $3.24-0.66 \mu \mathrm{g} / \mathrm{ml}$ of rhodamine $\left(\mathrm{r}^{2}=0.9984\right)$ for analyses in PBS pH 7.4; (ii) 7.05-0.56 $\mu \mathrm{g} / \mathrm{ml}$ of rhodamine $\left(\mathrm{r}^{2}=0.9991\right)$ for analyses in PBS $\mathrm{pH}$ 5.8.

\section{In vivo experiments}

All the in vivo experiments were performed following the Guidelines for Animal Care and Use of the National Institutes of Health. The study was approved by Italian Ministry of Health (permit number 183). Two lots of fifteen adult male rats weighing 200-220g were acclimatized for one week before the study with free access to water and food. The rats were anaesthetized with Zoletil 100 (100 mg/kg, i.p.) and placed on a heated working surface to prevent hypothermia. Both lots respectively received an IN injection of $50 \mu \mathrm{l}$ of positive or negative rhodamine labeled NPs. The IN injection was given according to Dyer et al. (2002). Briefly, the rats were placed in a supine position and $25 \mu \mathrm{l}$ were administered in each opening nostril (alternating) using a microliter syringe attached, via a needle, to a short polyethylene tubing, inserted approximately $0.7 \mathrm{~cm}$ into one nostril. The procedure was performed slowly in about 1 minute. Each lot was divided into three groups of five rats each and the three groups were sacrificed at different time intervals $(8,24,48 \mathrm{~h})$ and no treat. A control group of five rats was treated with $25 \mu 1$ of rhodamine B solution in each nostril. The animals were deeply anaesthetized with an intraperitoneal injection of Zoletil 100 (100 $\mathrm{mg} / \mathrm{kg}$ ) and Dexdomitor $(20-30 \mu \mathrm{g} / \mathrm{Kg})$ and perfused trancardially with a $4 \%$ paraformaldehyde (PFA) solution in $0.1 \mathrm{M}$ phosphate buffer solution (PBS, pH 7.4). The brains were removed, postfixed overnight in the same 4\% PFA and then transferred into a 30\% sucrose cryoprotective solution in PBS at $4{ }^{\circ} \mathrm{C}$ for2-3 days. Serial $25 \mu \mathrm{m}$ frozen sections of the brain were cut along the sagittal plane, mounted on slides, and air-dried. The fluorescence was examined on a fluorescence 
microscope (Nikon Eclipse 80i) equipped with filters for the visualization of rhodamine (excitation $530-560 \mathrm{~nm}$ ). Images were captured using a digital camera (Nikon) and adjusted for contrast with Adobe Photoshop without compromising data integrity.

\section{Results}

The hydrodynamic diameter is one of the most important parameters for the development of suitable nanocarriers for nose to brain (N2B) delivery. Only small particles can be transported to the brain via the olfactory or the trigeminal nerves (Mistry et al., 2009, Mistry et al. 2015). PLGA was chosen as a primary polymer to prepare negative surface nanoparticles. PLGA is widely used as a suitable polymer to prepare nanocarriers and it is a well-known material for our research group (Li Volti et al., 2012; Vicari et al., 2008). Chitosan was chosen to modify PLGA nanoparticles and to obtain positive nanoparticles in order to compare two nanosystems having opposite surface charge. The influence of different PLGA/CS ratios on the physico-chemical characteristics of colloidal carriers was evaluated to select the nanosuspension with the most suitable properties in order to perform in vivo studies for N2B delivery.

Microparticles could release molecules in the nasal cavity determining diffusion of free molecules to the brain. Migration may occur through the nasal olfactory and respiratory mucosa, as well as through the capillaries, lymphatic, and cerebrospinal fluid present in the nasal mucosa (Rassuet al., 2015). Once released in the nasal cavity, the free drug can be affected by biological environment degradation and the drug therapeutic concentration may consequently be achieved. Nanoparticles can be transported via olfactory epithelium and/or trigeminal nerve system and the encapsulated molecule can be released directly to the brain (Kozlovskaya et al., 2014; Mistry et al., 2015).

\section{The effect of polymer amount on mean size, surface properties and stability of CS/PLGANPs}

The nanoprecipitation method was successfully applied to prepare CS/PLGANPs. Nanocarriers were obtained through the precipitation of PLGA in the CS polyelectrolyte solution which 
introduces positive charges on the NPs, allowing us to obtain NPs with a mean size ranging from 200 to $800 \mathrm{~nm}$ and a polydispersity index ranging from 0.2 to 0.4 . As shown in figure 1 , only the formulation having the higher level of polymer produced a heterogeneous particle population. NPs smaller than $250 \mathrm{~nm}$ in diameter were obtained at a low polymer concentration (from 3 to $5 \mathrm{mg} / \mathrm{ml}$ ). All the nanocarriers presented a positive charge about $+70 \mathrm{mV}$ and the values were not influenced by PLGA amount (data not reported).

Figure 1 should be inserted here

To support PCS results even Turbiscan analysis was performed. We determined the nano-dispersion stability as a function of PLGA amount. NPs were analyzed immediately after preparation with an appropriate dilution. Taking into account that the nanosuspensions were destabilized through a sedimentation and/or aggregation phenomenon, TSI (Turbiscan Stability Index) was examined. Figure 2A showed the TSI values for all the nanosuspensions at $25^{\circ}$ for up to $48 \mathrm{~h}$. The nanosuspension with the lowest polymer amount is the most stable nanoparticle formulation (lowest values of TSI). In figure $2 \mathrm{~B}, \Delta$ Transmission $(\Delta \mathrm{T})$ and $\Delta$ Backscattering $(\Delta \mathrm{Bs})$ data showed no variations in the middle part of the graph, so no aggregation occurred. The variation at the bottom may represent a sedimentation phenomenon of a re-dispersible nanosuspension. All NPs appeared sufficiently stable for up to 48 according to the high values of zeta potential.

Figure 2 should be inserted here

\section{The properties of positive and negative rhodamine labeled NPs}

We focused our attention on two types of polymeric NPs for in vivo studies, in order to investigate their fate in the sub-regions of the brain after IN administration in healthy rats.

NPs were characterized using thermal analysis, which represents a useful technique to detect NP properties after the production process (Gill et al., 2010). DSC thermograms of the raw materials and selected NPs are shown in Figure 3. PLGA sample (C) presented a glass transition temperature ( $\mathrm{T}_{\mathrm{g}}$ ) of about $40{ }^{\circ} \mathrm{C}$. Chitosan (D) presented an exothermic peak at $40^{\circ} \mathrm{C}$ due to the loss of water 
molecules. In the thermograms obtained through PLGA NPs (B) the vitreous transition is also present. After the addition of chitosan, the exothermic peak of NPBC3 (A) disappeared and the $\mathrm{T}_{\mathrm{g}}$ is rather weak (Latif et al., 2015).

Figure 3 should be inserted here

As depicted in the SEM images (figure 4 A and B), both nanosuspensions are spherical and with smooth surfaces. While NPA appeared well separated, NPBC3 presented some aggregates confirming the different values of P.D.I.

In figure 4 physico-chemical properties are also shown. Both nanosuspensions presented mean size $\leq 230 \mathrm{~nm}$ (figure $4 \mathrm{C}$ and D) with positive surface charge for CS/PLGA NPs and negative for PLGA NPs (figure $4 \mathrm{E}$ and $\mathrm{F}$ ).

The mean size distribution (PDI), as also shown by SEM, highlight a more homogeneous particle population for NPA respect to NPBC3.

Figure 4 should be inserted here

Even a rhodamine in vitro release study was performed in order to evaluate that the fluorescence observed through microscopy analysis is due to the probe loaded into the nanocarriers and/or to the probe released from the nanocarriers (figure 1, supplementary data). As shown, 5\% of rhodamine was released after $24 \mathrm{~h}$; a slow and prolonged release was observed for up to $48 \mathrm{~h}$ where $15 \%$ of rhodamine was released (maximum in vivo experiment time).

\section{Sub-region localization in the rat brain of fluorescent labeled polymeric NPs}

The localization of negative surface charge (NPA) and positive surface charge (NPBC3) NPs were investigated in brain sub-regions at 8, 24 and $48 \mathrm{~h}$ after IN administration. We investigated the brain sub-regions in rats that are the principle targets for the different neurological diseases (figure 2, supplementary). The sections were prepared according to the sagittal illustration of the rat brain in 
Paxinos and Watson (Paxinos, 1982). Preliminary study demonstrated that no or only very few cells presented fluorescence after 2 and 4 hours of the nasal administrations (data not reported).

No fluorescence is depicted in non-treated brain rats (figure 5A) and slight and homogenous rhodamine distribution is shown in rat treated with rhodamine solution (figure 5B). The uptake of NPs in the brain was significantly increased 8-24 and 48 hours after IN administration and the labeled NPs were found in some brain areas. All NPs were prevalently localized in the cytosol of neural cells (figure 5C). NPs were also observed outside the cells, but were found with reduced frequency when compared to the level found in the cytosol.

Figure 5 should be inserted here

A summary of the labeling found in the various brain regions with negative and positive NPs was reported in Table 1.

Table 1 should be inserted here

The areas, where we found the NPs localized, are the following: Cerebral cortex, the frontal and parietal cortex, prevalently, while only a few NPs were found in the occipital cortex. The cerebral layers involved in NPs distribution were layer V and only a few in layers III, IV, VI and none in the superficial layers; Basal ganglia, in the caudate-putamen nucleus, prevalently; Hippocampus, in CA1-CA3 regions and in the granular layer of the dentate gyrus, few NPs were also observed in the entorhinal cortex and subiculum; Basal forebrain, the fluorescent label was observed in Piriform cortex and amigdaloid nuclei. In the Thalamus NPs were found in the reticular nucleus, central complex, anterior and dorsal nuclei; Mesencephalon, in the tegmental and pretectal nuclei. In the Cerebellum NPs were found in the Purkinje cell as well as in the cerebellar and vestibular nuclei; Myelencephalon, the reticular substantia, as well as spinal, trigeminal and facial nuclei. In the other brain sub-regions, here not mentioned, no or very scant negative and positive NPs were found (data not shown). The difference in fluorescence signals between the in vivo micrographs of the two type of NPs is a clear demonstration that is not due to the free marker. 
Regarding the trend of each nanosuspension we could describe a localization time - dependent on the surface charge of NPs. In fact, after $8 \mathrm{~h}$ of IN administration a decrease in fluorescence for negative NPs from the rostral to caudal area was found. An opposite trend was observed after $48 \mathrm{~h}$, where the caudal region showed a higher fluorescence compared to the caudal, while after $24 \mathrm{~h}$ a uniform distribution in all the cerebral structures was found (figure 6). The localization of CS/PLGA NPs had a different trend compared to negative NPs (figure 7). Since we found a weak labeling in all areas after $8 \mathrm{~h}$ and $24 \mathrm{~h}$ of IN administration, except in the cerebral cortex where the signal was moderate after $24 \mathrm{~h}$, only after $48 \mathrm{~h}$ did we observe an intense/moderate labeling in some cerebral structures.

Figures 6 and 7 should be inserted here

\section{Discussion}

Preliminary screening is important to select the ideal carrier to perform an in vivo study. According to Sanna et al. (2012), the combination of PLGA with chitosan leads to an increase of NP mean size strictly correlated with the increment of PLGA concentration. As shown in figure 1, the increase in polymer concentrations significantly increases the mean particles size. This variable did not influence the surface charge values largely governed by chitosan adsorption on the surface of the NPs. In this work we demonstrate that a reduction of the ratio between PLGA and CS used for the preparation of NPs, allows for the formation of a nanocarrier with a positively charged surface and a mean size below $250 \mathrm{~nm}$. A positive charge of NPBC NP series is due to the protonation of amine groups of CS, the possible electrostatic interaction with carboxylic groups of PLGA masking negative charges. Zeta potential is an important parameter because it allows for the prediction of physical stability and, according to the "electrostatic theory of mucoadhesion", even nanosphere/IN mucoadhesion to increase residence time (Shaikh et al., 2011). The highest Zeta potential values, either positive or negative in absolute value, produce stable dispersions due to particle electrostatic repulsions. The electrostatic repulsions between particles with the same electrical charge prevent 
particle aggregation. The highly positive Zeta potential has the potential to produce a formulation with good stability (Honary et al., 2013b). Turbiscan and PCS taken together allowed us to select the NPBC3 sample for the following investigations. NPBC NP series were stable against sedimentation, as evaluated by the Turbiscan analysis. NPBC3 demonstrated a good stability for the total duration of the experiment compared to the other samples, as elucidated by TSI values (figure 2A). $\Delta \mathrm{T}$ and $\Delta \mathrm{B}$ data showed no variations in the middle part of the graph, which means there was no flocculation/aggregation of the sample, thus confirming its higher stability in terms of particle size. As previously observed by PCS, this phenomenon is correlated with high zeta potential value (Bru et al., 2004). NPBC3 nanosuspension is affected by the sedimentation process; in fact, the increase of backscattering level that occurred at the bottom indicates a decrease in the concentration of particles consecutive to the sediment formation. The obtained cake was easily re-dispersible (Dihang et al., 2005).

NPBC3 was selected as the positive formulation to perform in vivo studies along with NPA (negative). The NP samples selected (NPA and NPBC3) were further characterized (figure 3 and figure 4). Figure 4 summarizes some physico-chemical properties of both nanocarriers. Slight morphological differences were observed between the two samples (figure 4, A and B). The aggregate particles observed through SEM are due to the dried process necessary to perform analyses.

In order to detect the location/distribution in the brain, rhodamine was encapsulated into the nanocarriers as a fluorescent marker. Moreover, cellular uptake of both NPs was investigated. For both NPs the encapsulation efficiency (E.E.) of rhodamine was assessed and it resulted in $\sim 10 \%$. The selected nanocarriers showed a mean size $<250 \mathrm{~nm}$ and opposite surface charge.

As previously reported by Mistry et al. (2015), there are three routes that allow NPs to achieve brain delivery after IN administration (systemic, olfactory and trigeminal pathway). We could consider nose to brain transport when the amount of NPs that reaches the brain through the systemic pathway 
is negligible when compared to trigeminal and olfactory pathways. In the last two years, several authors have examined the influence of NP surface chemistry on the biological environment, in order to increase the therapeutic efficacy of NPs (Honary et al., 2013a; De Jong et al., 2008).

However, there are relatively few studies that describe the influence of the surface charge of NPs on the localization in different brain sub-regions (rostral and/or caudal brain regions) during a time course after IN administration (Buchner et al., 1987). Knowing where and when NPs arrive in the brain is very important for drug delivery. Mechanisms by which NPs internalize and transport into the brain are poorly defined. The interaction of particles with cells is known to be strongly influenced by particle size, but little is known about the interdependent role that size, shape, and surface chemistry have on cellular internalization and intracellular trafficking of micro- and nanoparticles.

NPs mean diameter affects their interactions with cells and determines the active or passive cellular internalization and intracellular localization. Once in the body NPs are selectively transported into and out of the cells via endocytosis and exocytosis mechanisms. Following exocytosis NPs may have the ability to pass through in vivo barriers and cause unpredicted biological effects. The mean size itself of particles can determine the pathway of intracellular entry (Shang et al., 2014). Different mechanisms of endocytosis can be involved, varying with the size of the transport vesicles and the cargo properties. The endocytosis pathways are typically classified into clathrin- and caveolae-mediated endocytosis, phagocytosis, macropinocytosis, and pinocytosis (Oh et al., 2014).

Many types of cells use the clathrin- and caveolae-mediated endocytosis pathways to internalize nanoscale materials, including NPs.

Clathrin- and caveolae-mediated endocytosis indicates receptor-mediated endocytosis. Macropinocytosis is a clathrin-, caveolin-, and dynamin-independent process that involves uptake of a larger volume of the membrane allowing particles of sizes $\approx 1 \mu \mathrm{m}$ to be internalized. Similar to macropinocytosis, phagocytosis is also a clathrin-, caveolin-, and dynamin-independent process and 
is proficient in internalizing larger particles including pathogens (Jiang W et al., 2008; Geiser M. 2010). The physical size would not be fully reflected when they meet the plasma membrane of the cell. Most NPs tend to aggregate in biological solutions, increasing their overall size. Likewise, considering the possibility of the aggregation of NPs, the size of an aggregated nanocluster is larger than a single NP and will affect the internalization rate accordingly. The patterns of cellular uptake could also vary according to nanoparticle material type.

Taking into account some results obtained by other authors NPs displayed non-specificity of endocytosis routes correlated with their mean diameter. In an in vitro study undertaken by Nicolete et al., PLGA microparticles $(6.5 \pm 3.9 \mu \mathrm{m})$ were still attached to the cell surface and required more time for endocytosis to occur. Simultaneously, PLGA NPs of size $389 \mathrm{~nm}$ (PDI =0.2) had already, within the same time period, been encapsulated in vesicles and endocytosed into the intracellular compartment (Nicolete R, et al., 2011). These results were consistent with the study conducted by Loh et al., which demonstrated poor internalization capability of chitosan particles that were $1 \mu \mathrm{m}$, much unlike the extensive uptake of NPs of sizes 110-390 nm (Loh JW, et al., 2012). Moreover, as demonstrated by Lu et al., it seems that the internalization of nanoparticles with a diameter less than $200 \mathrm{~nm}$ involves clathrin-coated pits. With size increasing, a shift to a mechanism that relies on caveolae-mediated internalization becomes apparent, which becomes the predominant pathway of entry for particles of $500 \mathrm{~nm}$ in size (Lu F, et al., 2009). So, we could hypothesized that for both our type of NPs the same internalization mechanism occurred, related to the range of mean size.

In addition to particle size, shape is another essential property of nanoparticles that plays important role in various biological processes and in vivo behavior. NPs shape shown to have a profound effect on the transport through the vasculature, circulation half-life, targeting efficiency, phagocytosis and endocytosis. It seems that the shape that contribute to phagocytosis inhibition and long circulation will, in turn, impede the endocytosis of NPs by the targeted cells (Duan et al., 2013). 
Both nanosuspensions, (NPA, NPBC3), are spherical, with smooth surfaces and rigid (not deformable). Non-spherical NPs show superior properties compared to spherical NPs in terms of escape from phagocytosis, long circulation half-life, and firm binding to the target tissues, however, the uptake of NPs significantly increase with increasing spherical morphology.

Overall, this study demonstrates that nanoparticle charge is a crucial characteristic in terms of dictating their distribution and translocation in both rostral and/or caudal brain regions. Our in vivo experiments demonstrate that the fate of negative and positive NPs in neural cells was strictly correlated with their surface properties. Our data could not exclude the involvement of the systemic pathway. The localization of NPs in some brain sub-regions after $8 \mathrm{~h}$ suggested the occurrence of a probable direct N2B transfer.

In fact, no PEGylated NPs are removed through a mononuclear phagocyte system (MPS) after 4/6 hours of their presence in the body (Suk et al., 2016). Besides, NPs without suitable ligands on their surface are not able to cross the blood brain barrier (Tosi et al., 2016).

At this point the question is: why did NPs arrive in the brain after a lag time, and different subregions are involved? The answer is important not only to explain the potential application of nanocarriers but also the implication in the toxicological phenomenon of these colloidal carriers.

First of all, to better understand our statements, it is important to introduce some results obtained by other authors in this field and the possible mechanisms involved. Some authors described the pathways and mechanism by which free molecules pass the nasal region to reach the brain if not absorbed via the systemic pathway and not drained by nasal lymphatic (Lockhead et al., 2012). There are two possibilities: the drug is transported to the brain encapsulated in the NPs through the neural or trans-epithelial pathways or the drug is released on the surface of the nasal epithelium or inside the tissue and then transported across the membrane (Mistry et al., 2009). Some experimental evidence demonstrated that the pathways involved nerves (olfactory and trigeminal) connecting the nasal passages to the brain and spinal cord (Dhuria et al., 2010). The nanoparticles could be taken up by the neuronal cells by processes of endocytosis, i.e., micropinocytosis or phagocytosis. Only 
nanoparticles with a diameter smaller than $20 \mathrm{~nm}$ can achieve extracellular transport from the nasal cavity to the brain, only nanoparticles with diameter smaller of olfactory axons can be intracellularly transported to the bran via the olfactory neural pathway. We exclude the involvement of the extracellular pathway via tight junctions since the NPs were too large to pass through tissue using this route (Mistry et al., 2015).

Analyzing our data, we could hypothesize that negative NPs arrived at the rostral sub-regions and then they were transported to caudal regions. This transport involved subcortical areas. Positive CS/PLGANPs were slower in transferring to the brain parenchyma than negative NPs and presented a prevalent localization in the caudal sub-regions. Two different pathways were used by the two types of NPs tested an intra-neuronal pathway for positive NPs, because the intra-neuronal pathway involves axonal transport and requires hours to days for drugs (and assumed nanoparticles) to reach brain regions. While the negative NPs may reach the brain areas through the extra-neuronal pathway, which probably relies on bulk flow transport through peri-neural channels that deliver drugs/particles directly to the brain parenchymal tissue and/or cerebral spinal fluid (CSF) (Chen et al., 2012). Therefore, we suggested that the surface differences between the nanocarriers tested, deeply influence the time course. In particular, we could affirm that chitosan enhanced the NPs residence time, due to electrostatic interactions and, at the same time for the same reason, it increased the time to reach the brain for NPs.

The appearance of the fluorescence signal in rostral brain regions at early time points for negative NPs suggested the olfactory transport considering that the olfactory pathway may provide both intra-neuronal and extra-neuronal access to the brain (Hadaczek et al., 2006); on the contrary, the transport of positive NPs was different, since we found a weak fluorescent signal in all areas at early time points and a strong fluorescence intensity in caudal brain after $48 \mathrm{~h}$ suggesting the involvement of the trigeminal nerve transport for positive NPs.

Furthermore, the diameter and shape of these carrier system also play an essential for their cellular uptake. 


\section{Conclusion}

In this work nanotechnology systems and nose-to-brain delivery were investigated in order to provide new, patient-friendly solutions to delivering therapeutic agents to the brain. A specific ratio between PLGA and CS is needed to obtain a carrier having good stability at $25^{\circ} \mathrm{C}$. Our data demonstrated that NPBC3 is the more suitable positive nanocarriers to perform in vivo study comparing its localization in respect to NPA (negative nanocarrier). Surface charge is a critical parameter to determine the site of localization of NPs. Our in vivo studies demonstrated that both NPs can reach the brain, even if the uptake of negative carriers seems prevalent in rostral subregions, suggesting that NPA were possibly transported via olfactory pathway while NPBC3 seems to involve the trigeminal pathway.

Our data also demonstrated differences in the localization of NPs in the brain areas dependent on the NPs physico-chemical properties in function at the time after IN administration. These findings raised the hypothesis that therapeutic agent loaded NPs may have a direct access to the CNS following IN administration.

Not only NPs can reach the brain but it is possible to obtain a prevalent localization in caudal or rostral area depending on the NP surface charge. The results observed suggested that not only the mean size, as previously demonstrated by other authors (Mistry et al, 2015), is an important parameter but also the surface properties. On the basis of the neurological disease and in order to obtain a prevalent localization in the brain region affected, the accurate selection of the polymeric matrix to formulate nanocarriers is important for influencing surface charge.

\section{Contributors}

All authors gave substantial contributions to the conception or design of the work. In particular, Teresa Musumeci, the corresponding author, Serapide Francesca and Rosalia Pellitteri participated in the acquisition, analysis, or interpretation of data. Angela Bonaccorso participated in the 
development of experimental section. All authors participated in the drafting of the work and the critical revision of its content. All authors approved the final version to be published.

\section{Acknowledgements}

This work was supported by the Italian grant number PRIN 2010/2011 [grant number: 2010H834LS_005] and POR.FESR titled “Piattaforma regionale translazionale per la salute" [CUP: B65E12000570008]. We are grateful to Dr. Elisa Giuffrida for her collaboration during the in vivo studies.

Dr. Angela Bonaccorso was supported by the International PhD program in Neuroscience, University of Catania, Italy.

\section{References}

Bharali, D.J., Armstrong D., Mousa, S.A. 2013. Hybrid polymeric nanoparticles: potential candidate for ophthalmic delivery. Methods Mol. Biol.1028:279-86. DOI: 10.1007/978-1-62703-475-3_18.

Bru, P., Brunel, L., Buron, H, Cayré, I., Ducarre, X., Fraux, A., Mengual, O., Meunier, G., de Sainte Marie A. 2004.Particle size and rapid stability analyses of concentrated dispersions: Use of multiple light scattering inT. Provder and J. Texter, Particle Sizing and Characterization. ACS Symposium Series- 881: 45-60. Chapter DOI: 10.1021/bk-2004-0881.ch003.

Buchner, K., Seitz-Tutter, D., Schonitzer, K., Weiss, D.G.1987. A quantitative study of anterograde and retrograde axonal transport of exogenous proteins in olfactory. Neuroscience. 22:697-707.

Budhiana, A., Siegelb, S. J., Wineya,K. I.2008. Controlling the in vitro release profiles for a system of haloperidol-loaded PLGA nanoparticles. Int. J. Pharm. 346:151-159. DOI: 10.1016/j.ijpharm.2007.06.011 
Casettari, L, Illum, L. 2014. Chitosan in nasal delivery systems for therapeutic drugs. J Control Release.2014; 190:189-200. DOI: 10.1016/j.jconrel.2014.05.003.

Chapman, C.D., Frey, W.H., Craft, S., Danielyan, L., Hallschmid, M., Schiöth, H.B., Benedict, C.2013. Intranasal treatment of central nervous system dysfunction in humans. Pharm. Res.Disordr. 30: 2475-84. DOI: 10.1007/s11095-012-0915-1.

Charlton, S. T., Davis, S. S., Illum, L.2007. Evaluation of bioadhesive polymers as delivery systems for nose delivery: In vitro characterisation studies. J. Controlled Release. 2007; 118:225-234. DOI:10.1016/j.jconrel.2006.12.014.

Chen, J., Zhang, C., Liu, Q., Shao, X., Feng, C., Shen, Y., Zhang, Q., Jiang, X.2012. Solanum tuberosum lectin-conjugated PLGA nanoparticles for nose-to-brain delivery: in vivo and in vitro evaluations. J. Drug Target. 2012; 20: 174-184. DOI: 10.3109/1061186X.2011.622396.

De Jong, W. H., Borm, P.J.2008.Drug delivery and nanoparticles: Applications and hazards. Int. J. Nanomedicine. 3:133-149.

Dhuria, S. V., Hanson, L. R., Frey IIW. H. 2010.Intranasal delivery to the central nervous system: Mechanisms and experimental considerations. J. Pharm Sci. 4:99:1654-1673. DOI: 10.1002/jps.21924.

Dihang, H., Bru, P., Meunier, G., Pouligny, B., Snabre,P. 2005. Effect of centrifugation on sample homogeneity. Application paper. 2005; 1-5. 
Djupesland, P. G., Messina, J. C., Mahmoud,R. A. 2014.The nasal approach to delivering treatment for brain deseases: an anatomic, physiologic, and delivery technology overview. Ther. Deliv. 5:709733. DOI: $10.4155 /$ tde. 14.41.

Duan X, LiY. Physicochemical Characteristics of Nanoparticles Affect Circulation, Biodistribution, Cellular Internalization, and Trafficking. Small. 2013; 9(9-10):1521-32. DOI: $10.1002 / \mathrm{smll} .201201390$

Duttagupta, D.S., Varsha, M. J., Vilasrao,J. K. 2015. Chitosan: A Propitious Biopolymer for Drug Delivery. Curr. Drug Deliv. 12:369-381.

Dyer, A.M., Hinchcliffe, M., Watts, P., Castile, J., Jabbal-Gill, I., Nankervis, R., Smith, A., Illum, L.2002. Nasal delivery of insulin using novel chitosan based formulations: a comparative study in two animal models between simple chitosan formulations and chitosan nanoparticles. Pharm Res. 19:998-1008.

Geiser M. Update on macrophage clearance of inhaled micro- and nanoparticles. J Aerosol Med Pulm Drug Deliv. 2010;23(4):207-17. DOI: 10.1089/jamp.2009.0797.

Gill, P., Moghadam, T. T., Ranjbar,B. 2010. Differential Scanning Calorimetry Techniques: Applications in Biology and Nanoscience. J Biomol Tech.21:167-193.

Hadaczek P, Forsayeth J, BankiewiczK.2006. Delivery of molecular therapeutics into the CNS and their distribution within the brain, in: During, M.J. and Kaplitt M.G.(ed), Gene Therapy of the Central Nervous System: From Bench to Bedside. Michael G. Kaplitt.(academic press) 121-129. 
Honary, S., Zahir, F.2013a.Effect of Zeta Potential on the Properties of Nano-Drug Delivery Systems -A Review (Part 1).Trop J Pharm Res. 2013; 12 (2): 255-264.

Honary, S., Zahir, F.2013b.Effect of Zeta Potential on the Properties of Nano-Drug-Delivery Systems (Part 2). Trop J Pharm Res. 12:265-273.

Jiang W, Kim BY, Rutka JT, Chan WC. Nanoparticle-mediated cellular response is size-dependent. Nat Nanotechnol. 2008;3:145-150. DOI: 10.1038/nnano.2008.30.

Kapoor, D.N., Bhatia, A., Kaur, R., Sharma, R., Kaur, G., Dhawan S.2015. PLGA: a unique polymer for drug delivery. Ther Deliv. 6:41-58. DOI: 10.4155/tde.14.91.

Kozlovskaya, L., Abou-Kaoud, M., Stepensky, D. 2014. Quantitative analysis of drug delivery to the brain via nasal route. J Control Release.189:133-140. DOI: 10.1016/j.jconrel.2014.06.053.

Kumari A., Kumar S., Subhash Y., Yadav C. 2010. Biodegradable polymeric nanoparticles based drug delivery systems. Colloids and Surfaces B: Biointerfaces. 75:1-18.

Latif, A., N. A., Awad, H. M., Mouneir S. M. and Elnashar, M. M. 2015. Chitosan-benzofuran adduct for potential biomedical applications: Improved antibacterial and antifungal properties. Pharm. Lett.; 7:107-117.

Li Volti, G., Musumeci, T., Pignatello R., Murabito, P., Barbagallo, I., Carbone, C., Gullo, A., Puglisi, G. 2012.Antioxidant potential of different melatonin-loaded nanomedicines in an experimental model of sepsis.Exp Biol Med. 237:670-7. DOI: 10.1258/ebm.2012.011425. 
Lockhead, J. J., Thorne,R. G. 2012. Intranasal delivery of biologics to the central nervous system. Adv. Drug Deliver. Rev. 64:614-628. DOI: 10.1016/j.addr.2011.11.002.

Loh JW, Saunders M, Lim LY. Cytotoxicity of monodispersed chitosan nanoparticles against the Caco-2 cells. Toxicol Appl Pharmacol. 2012;262(3):273-282. DOI: 10.1016/j.taap.2012.04.037.

Lu F, Wu SH, Hung Y, Mou CY. Size effect on cell uptake in wellsuspended,uniform mesoporous silica nanoparticles. Small. 2009;5(12):1408-13. DOI: 10.1002/smll.200900005.

Mistry, A., Stolnik, S., Illum, L.2009. Nanoparticles for direct nose-to-brain delivery of drugs. Int. J. Pharm. 2009; 379:146-157. DOI: 10.1016/j.ijpharm.2009.06.019.

Mistry A, Stolnik, S., Illum L.2015. Nose-to-Brain Delivery: Investigation of the Transport of Nanoparticles with Different Surface Characteristics and Sizes in Excised Porcine Olfactory Epithelium. Mol. Pharmaceut.12:2755-2766. DOI: 10.1021/acs.molpharmaceut.5b00088.

Meredith M. E., Salameh T. S., and Banks W. A. 2015. Intranasal Delivery of Proteins and Peptides in the Treatment of Neurodegenerative Diseases. The AAPS Journal, 17(4):780-788.

Musumeci, T., Bucolo, C., Carbone, C., Pignatello, R., Drago, F., Puglisi, G. 2013.Polymeric nanoparticles augment the ocular hypotensive effect of melatonin in rabbits. Int. J. Pharmceut. 440:135-40. DOI: 10.1016/j.ijpharm.2012.10.014.

Musumeci, T., Pellitteri, R., Spatuzza, M., Puglisi, G. 2014. Nose-to-Brain Delivery: Evaluation of Polymeric Nanoparticles. J Pharm Sci. 103:628-635. DOI: 10.1002/jps.23836. 
Nicolete R, Dos Santos DF, Faccioli LH. The uptake of PLGA micro or nanoparticles by macrophages provokes distinct in vitro inflammatory response. Int Immunopharmacol. 2011;11(10):1557-1563. DOI: 10.1016/j.intimp.2011.05.014.

Oh N, Park JH. Endocytosis and exocytosis of nanoparticles in mammalian cells. Int J Nanomedicine. 2014;9 Suppl 1:51-63. DOI: 10.2147/IJN.S26592. eCollection 2014.

Paxinos G., Watson C. The rat brain in stereotaxic coordinates. Academic press, 1982.

Phukan, K., Nandy, M., Sharma, R.B., Sharma, H.K.2016. Nanosized Drug Delivery Systems for Direct Nose to Brain Targeting: A Review. Recent Pat Drug Deliv Formul.10(2):156-64.

Piazza, J., Hoare, T., Molinaro, L., Terpstra, K., Bhandari, J., Selvaganapathy, P. R, Gupta, B., Mishra, R. K. 2014.Haloperidol-loaded intranasally administered lectin functionalized poly(ethylene glycol)-block-poly(D,L)-lactic-co-glycolic acid (PEG-PLGA)nanoparticles for the treatment of schizophrenia.Eur J Pharm Biopharm. 87:30-39. DOI: 10.1016/j.ejpb.2014.02.007.

Rassu, G., Soddu, E., Cossu, M., Brundu, A., Cerri, G., Marchetti N., Ferraro L., Regan R.F., Giunchedi P., Gavini E., Dalpiaz A. 2015. Solid microparticles based on chitosan or methyl- $\beta$ cyclodextrin: a first formulative approach to increase the nose-to-brain transport of deferoxaminemesylate. J Control Release. 201:68-77. DOI: 10.1016/j.jconrel.2015.01.025.

Sanna, V., Roggio, A. M., Siliani, S., Piccinini, M., Marceddu, S., Mariani, A., Sechi,M.2012. Development of novel cationic chitosan and anionic alginate-coated poly(d,l-lactide-coglycolide) nanoparticles for controlled release and light protection of resveratrol. Int J Nanomed. 7:5501-5516. DOI: $10.2147 /$ IJN.S36684. 
Shadab Md, Shadabul Haque, Mohammad Fazil, Manish Kumar, Sanjula Baboota, Jasjeet K Sahni \& Javed Ali. 2014. Optimised nanoformulation of bromocriptine for direct nose-to brain delivery: biodistribution, pharmacokinetic and dopamine estimation by ultra-HPLC/mass spectrometry method. Expert Opin. Drug Deliv. 11(6):827-842.

Shaikh, R., Raghu, T., Singh, R., Garland, M. J., Woolfson, A. D., Donnelly,R. F. 2011.Mucoadhesive drug delivery systems. J Pharm Bioallied Sci. 3:89-100. DOI: 10.4103/09757406.76478.

Shang L, Nienhaus K and Nienhaus GU. Engineered nanoparticles interacting with cells: size matters. J Nanobiotechnology. 2014;12:5. DOI: 10.1186/1477-3155-12-5.

Suk J.S., Xu Q., Kim N., Hanes J., Ensign L. M. 2016. PEGylation as a strategy for improving nanoparticle-based drug and gene delivery. Adv. Drug Del. Rev. 99:28-51.

Tosi, G., Musumeci, T., Ruozi, B., Carbone, C., Belletti, D., Pignatello, R., Vandelli, M. A., Puglisi, G. 2016. The "fate" of polymeric and lipid nanoparticles for brain delivery and targeting: Strategies and mechanism of blood-brain barrier crossing and trafficking into the central nervous system. $\mathbf{J}$ Drug Deliv Sci Tec. 32:66-76. DOI:10.1016/j.jddst.2015.07.007.

Vicari, L., Musumeci, T., Giannone, I., Adamo, L., Conticello, C., De Maria, R., Pignatello, R., Puglisi, G., Gulisano, M.Paclitaxel loading in PLGA nanospheres affected the in vitro drug cellaccumulation and antiproliferative activity. BMC Cancer. 2008;8:212. DOI: 10.1186/14712407-8-212. 
Van Berlo D., Hullmanna M., Wessels A., Scherbart A.M.,Cassee F.R., Gerlofs-Nijland M.E., Albrecht C., Schins R.P.F. 2014. Investigation of the effects of short-term inhalation of carbon nanoparticles on brains and lungs of c57bl/6j and p47phox-/- mice. NeuroToxicology. 43:65-72. DOI: 10.1016/j.neuro.2014.04.008.

Zhang C., Chen J., Feng C., Shao X., Liu Q., Zhang Q, Pang Z, Jiang X. 2014. Intranasal nanoparticles of basic fibroblast growth factor for brain delivery to treat Alzheimer's disease. Int. J. Pharm. 461(1-2):192-202. DOI: 10.1016/j.ijpharm.2013.11.049.

\section{Figure captions}

Graphical abstract: Schematic representation of rhodamine-labeled NPs distribution, 8, 24 and 48h after IN administration in rat. Red spot indicates NPA; Blue-red spot indicates NPBC3. The intense, moderate and weak labeling correspond to large, medium and small spots respectively. The brain areas investigated are: cerebral cortex (beige); basal ganglia (light blue); hyppocampus (green); thalamus (pink); mesencephalon (orange); cerebellar cortex and nuclei (violet); myelencephalon (yellow).

Figure 1. Mean size and polidispersitivity index of positive CS/PLGA nanoparticles obtained with a different amount of PLGA at an invariable amount of surfactant and chitosan. Values are presented as the mean \pm S.D. $(n=3)$.

Figure 2. Turbiscan Stability Index (A) as a function of an initial amount of PLGA polymer in

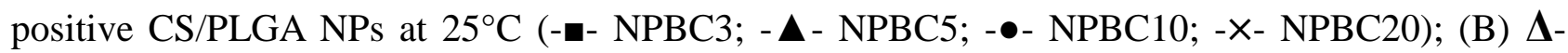
Backscattering $(\boldsymbol{\Delta B s})$ and $\boldsymbol{\Delta}$-Transmission $(\boldsymbol{\Delta T})$ of NPBC3 at $25{ }^{\circ} \mathrm{C}$.

Figure 3. DSC Thermograms of A) NPBC3 (CS/PLGA NPs); B) NPA (PLGA NPs); C) PLGA; D) chitosan. 
Figure 4. Physico-chemical and morphological properties of the rhodamine labeled nanocarriers (NPA and NPBC3) selected for in vivo studies. A) Scanning electron micrograph of NPBC3 (CS/PLGA NPs); B) Scanning electron micrograph of NPA (PLGA NPs); C) Mean size and PDI of NPBC3 (CS/PLGA NPs); D) Mean size and PDI of NPA (PLGA NPs); E) Zeta potential of NPBC3 (CS/PLGA NPs); F) Zeta potential of NPA (PLGA NPs).

Figure 5. A) Photograph of rat brain not treated; B) Photograph of rat brain treated with rhodamine B solution; C) Photograph of rhodamine labeled PLGA NPs into cytosol of neural cells of the rostral region of the brain after IN administration ( $24 \mathrm{~h})$.

Figure 6. Photomicrographs illustrating the localization of the NPA (negative NPs) in relation to the brain sub-regions during the time-course $(8,24,48 \mathrm{~h})$ after IN administration. Rostro-caudal sequence of the brain sub-regions investigated: Frontal cortex (FC); Basal Ganglia (BG); Hippocampus (HPC); Mesencephalon (MES); Cerebellar nuclei (CN); Myelencephalon (MYE). The white narrow indicates the presence of rhodamine-labeled NPs. Scale bar=50 $\mu \mathrm{m}$.

Figure 7. Photomicrographs illustrating the localization of the NPBC3 (positive NPs) in relation to the brain sub-regions during time-course $(8,24,48 \mathrm{~h})$ after IN administration. Rostro-caudal sequence of the brain sub-regions investigated. Frontal cortex (FC); Basal Ganglia (BG); Hippocampus (HPC); Mesencephalon (MES); Cerebellar nuclei (CN); Myelencephalon (MYE). The white narrow indicates the presence of rhodamine-labeled NPs. Scale bar=50 $\mu \mathrm{m}$.

\section{Supplementary data}

Figure 1. Release profile of rhodamine from negative and positive NPs in PBS pH 5.8 and pH 7.4 at $37^{\circ} \mathrm{C}$ until $48 \mathrm{~h}$.

Figure 2. Modified sagittal section of the rat brain according to Paxinos and Watson. 